September 7, 2018

LBL-36338

\title{
Possible Light U(1) Gauge Boson Coupled to Baryon Number
}

\author{
Christopher D. Carone and Hitoshi Murayamaif \\ Theoretical Physics Group \\ Lawrence Berkeley Laboratory \\ University of California \\ Berkeley, California 94720
}

\begin{abstract}
We discuss the phenomenology of a light $\mathrm{U}(1)$ gauge boson, $\gamma_{B}$, that couples only to baryon number. We assume that the new $\mathrm{U}(1)$ gauge symmetry is spontaneously broken and that the $\gamma_{B}$ mass is smaller than $m_{Z}$. Nevertheless, we show that the model survives the current experimental constraints. In addition, we argue that evidence for the existence of such a particle could be hidden in existing LEP and Tevatron data. We determine the allowed regions of the $m_{B}-\alpha_{B}$ plane, where $m_{B}$ is the $\gamma_{B}$ mass, and where $4 \pi \alpha_{B}$ is the squared gauge coupling. We point out that in some parts of the allowed parameter space our model can account for rapidity gap events in proton-antiproton scattering seen at the Fermilab Tevatron.
\end{abstract}

\footnotetext{
*This work was supported by the Director, Office of Energy Research, Office of High Energy and Nuclear Physics, Division of High Energy Physics of the U.S. Department of Energy under Contract DE-AC03-76SF00098.

${ }^{\dagger}$ On leave of absence from Department of Physics, Tohoku University, Sendai, 980 Japan.
} 


\section{Disclaimer}

This document was prepared as an account of work sponsored by the United States Government. While this document is believed to contain correct information, neither the United States Government nor any agency thereof, nor The Regents of the University of California, nor any of their employees, makes any warranty, express or implied, or assumes any legal liability or responsibility for the accuracy, completeness, or usefulness of any information, apparatus, product, or process disclosed, or represents that its use would not infringe privately owned rights. Reference herein to any specific commercial products process, or service by its trade name, trademark, manufacturer, or otherwise, does not necessarily constitute or imply its endorsement, recommendation, or favoring by the United States Government or any agency thereof, or The Regents of the University of California. The views and opinions of authors expressed herein do not necessarily state or reflect those of the United States Government or any agency thereof or The Regents of the University of California and shall not be used for advertising or product endorsement purposes.

Lawrence Berkeley Laboratory is an equal opportunity employer. 
The standard model possesses a number of global U(1) symmetries that are assumed to be accidental symmetries of the theory. Baryon number, and the three types of lepton number (associated with the electron, muon, and tau) generate these U(1) symmetries. It has been argued, however, that global symmetries should be broken by quantum gravity effects [四], with potentially disastrous consequences. Baryon number-violating operators generated at the Planck scale can lead to an unacceptably large proton decay rate, especially in some supersymmetric theories [2]. This problem can be avoided naturally if baryon number is taken instead to be a local symmetry. Moreover, it is not even clear whether global phase rotations are consistent with the basic premise of local field theory [3]. For these reasons, and at the very least for aesthetics, it is natural to wonder whether any of the global U(1) symmetries of the Standard Model can be promoted to gauge symmetries in a phenomenologically acceptable way.

In this letter, we will consider the consequences of gauging the U(1) symmetry generated by baryon number, $\mathrm{U}(1)_{B}$. We assume that the symmetry is spontaneously broken and that the corresponding gauge boson $\gamma_{B}$ develops a mass $m_{B}<m_{Z}$. Of course, in the minimal Standard Model we cannot gauge baryon number alone, because the resulting field theory would suffer from gauge anomalies. However, by adding a small number of new fermions (that we can make heavier than $m_{\text {top }}$ by an appropriate choice of Yukawa couplings), we can gauge $\mathrm{U}(1)_{B}$ in an anomaly-free way. Then, the main question of interest to us is whether the $\gamma_{B}$ boson could have evaded all the available direct or indirect means of detection. If we call the squared gauge coupling $4 \pi \alpha_{B}$, then we can determine what regions of the $m_{B}-\alpha_{B}$ plane are excluded by the current experimental constraints. Considering the assumed lightness of the $\gamma_{B}$ boson, our conclusions are somewhat surprising: there are relatively large regions of the $m_{B}-\alpha_{B}$ plane in which our model is phenomenologically allowed [四]. In addition, for some of the allowed choices of the $\gamma_{B}$ coupling and mass, our model can also account for the rapidity gap events observed at the Fermilab Tevatron [5].

We will first concern ourselves with the $\gamma_{B}$ phenomenology, and then present an example of a simple, anomaly-free model at the end. We will see that the $\gamma_{B}$ boson is elusive for some of the same reasons that it is difficult to detect a light gluino [6] or stop [7]. Since the $\gamma_{B}$ boson couples only 
to quarks, its most important effects can be expected in the same processes used in measuring the QCD coupling $\alpha_{s}$. Thus, we will determine the allowed regions of the $m_{B}-\alpha_{B}$ plane by considering the following observables: the $Z$ hadronic width, the $Z \rightarrow 3$ jet and $Z \rightarrow 4$ jet total cross sections, the di-jet invariant mass distribution in $Z \rightarrow 4$ jets, and the hadronic decay width of the $\Upsilon(1 S)$. We will concentrate mostly on the region where $m_{B} \gtrsim 10 \mathrm{GeV}$, and the $\gamma_{B}$ boson decays to $q \bar{q}$ with the width $\Gamma_{B}=N_{F} \alpha_{B} m_{B} / 9$. The more general case will be considered in a longer publication [8].

The $Z$ hadronic width. The $\gamma_{B}$ boson contributes to the $Z$ hadronic width at order $\alpha_{B}$ through (1) direct production $Z \rightarrow \bar{q} q \gamma_{B}$, and (2) the $Z \bar{q} q$ vertex correction. Writing these two contributions as $F_{1}$ and $F_{2}$, we find that the nonstandard contribution to the $Z$ hadronic width, $\Delta \Gamma$, is positive and given by

$$
\frac{\Delta \Gamma(Z \rightarrow \text { hadrons })}{\Gamma(Z \rightarrow q \bar{q})}=\frac{\alpha_{B}}{18 \pi}\left[F_{1}+F_{2}\right]
$$

where

$$
\begin{aligned}
F_{1}= & (1+\delta)^{2}\left[3 \ln \delta+(\ln \delta)^{2}\right]+5\left(1-\delta^{2}\right)-2 \delta \ln \delta \\
& -2(1+\delta)^{2}\left[\ln (1+\delta) \ln \delta+\operatorname{Li}_{2}\left(\frac{1}{1+\delta}\right)-\operatorname{Li}_{2}\left(\frac{\delta}{1+\delta}\right)\right], \\
F_{2}= & -2\left\{\frac{7}{4}+\delta+\left(\delta+\frac{3}{2}\right) \ln \delta\right. \\
& \left.+(1+\delta)^{2}\left[\operatorname{Li}_{2}\left(\frac{\delta}{1+\delta}\right)+\frac{1}{2} \ln ^{2}\left(\frac{\delta}{1+\delta}\right)-\frac{\pi^{2}}{6}\right]\right\} .
\end{aligned}
$$

Here $\operatorname{Li}_{2}(x)=-\int_{0}^{x} \frac{d t}{t} \ln (1-t)$ is the Spence function, and $\delta=m_{B}^{2} / m_{Z}^{2}$. We compare this result to the uncertainty in the experimentally measured $Z$ hadronic width corresponding to a two standard deviation uncertainty in the extracted value of $\alpha_{s}\left(m_{Z}\right)=0.124 \pm 0.0086$ [9]. As shown in Fig. 1, this roughly excludes the region of parameter space above $\alpha_{B} \approx 0.2$.

$Z \rightarrow$ jets. The $\gamma_{B}$ boson contributes to $Z$ decay to four jets, via $Z \rightarrow$ $\bar{q} q \gamma_{B}, \gamma_{B} \rightarrow \bar{q} q$. In doing our parton-level jet analysis, we adopt the JADE algorithm, in which we require jets $i$ and $j$ to be separated in phase space by $y_{i j} \equiv 2 E_{i} E_{j}\left(1-\cos \theta_{i j}\right) / m_{Z}^{2}>y_{\text {cut }}$, where $E_{i}$ and $E_{j}$ are the jet energies, and $\theta_{i j}$ is the angle between the jets. If any pair of jets has $y_{i j}<y_{\text {cut }}$, then these are combined into one jet, and the event instead contributes to the three-jet cross section. Since two of the jets originate from the $\gamma_{B}$, the total four-jet 
cross section as a function of $y_{\text {cut }}$ will drop off as $y_{\text {cut }}$ is taken to be greater than $m_{B}^{2} / m_{Z}^{2}$. The four-jet cross section is shown in Fig. 2 as a function of $y_{\text {cut }}$, normalized to the lowest order two-jet cross section $\sigma_{0}$, for $\alpha_{B}=0.1$ and for a range of $m_{B}$ [10. We compare our results to the experimental bounds on the fraction of all four-jet events that are four-quark jet events, $9.1 \%$ (95\% C.L.) with $y_{\text {cut }}=0.01$ [1]. Comparing the $\gamma_{B}$ contribution to $\sigma_{4} / \sigma_{0}$ at $y_{\text {cut }}=0.01$ to the expected four-jet QCD background $\left(\sigma_{4} / \sigma_{0}\right) \mathrm{QCD} \approx 0.2$ gives us the bound shown at the top of Fig. 1. For the most part, this excludes no new parameter space beyond the region already excluded by our analysis of the $Z$ hadronic width.

The events that are not counted as four-jet events contribute to the total three-jet cross section, in principle yielding some enhancement over the expected rate. However, given the large three-jet QCD background, the three-jet analysis will not yield a further constraint.

$D i$-jet invariant mass peak in $Z \rightarrow 4$ jets. The di-jet invariant mass $m_{j j}$ distribution in $Z$-decay has been studied in searches for charged Higgs pairs, associated light and heavy Higgs production in two-Higgs-doublet models, and excited quark pairs that decay via $q^{*} \rightarrow q g$ [12]. In these studies, peaks in the $m_{j j}$ distribution from both particles were required, so that the results are irrelevant to our problem. In principle, one can look for a peak in the $m_{j j}$ distribution without any other requirements, but then one must contend with a huge QCD background. We show the $m_{j j}$ distributions in Fig. 3 for various values of $m_{B}$, together with the QCD background. We chose $y_{\text {cut }}=0.04$ to optimize the signal for $m_{B}=20 \mathrm{GeV}$. It is clear that the signal is overwhelmed by the background. A distribution that is more sensitive to the $\gamma_{B}$, especially for $m_{B} \lesssim 30 \mathrm{GeV}$, is the distribution of the smallest invariant mass $m_{\min }^{2}$ among the six possible combinations in four-jet events. We show the distribution of $y_{\min }=m_{\min }^{2} / m_{Z}^{2}$ in Fig. 4. The background dominates the signal by more than a factor of 7 , even on the peak. Moreover, the peak will be further smeared by hadronization effects and the resolution of the hadron calorimetry. Therefore no practical constraint exists from the $m_{j j}$ distribution. The search for a peak in the $m_{j j}$ distribution at hadron colliders is probably hopeless, given the much larger backgrounds.

$\Upsilon(1 S)$ Decay. The decay of $\Upsilon(1 S)$ is another place to look for the effect of the $\gamma_{B}$ boson, through its contribution to $R_{\Upsilon}=\Gamma(\Upsilon \rightarrow$ hadrons $) / \Gamma(\Upsilon \rightarrow$ 
$\left.\mu^{+} \mu^{-}\right)$. The constraint that we obtain depends on whether $\gamma_{B}$ appears as a real particle in the final state (when $m_{B}<m_{\Upsilon}$ ) or not (when $m_{B}>m_{\Upsilon}$ ). In the case where $m_{B}>m_{\Upsilon}$, the most stringent constraint comes from the additional contribution to the $\Gamma(\Upsilon \rightarrow$ hadrons $)$ from $s$-channel exchange of the $\gamma_{B}$ boson. This additional contribution is

$$
\Delta R_{\Upsilon}=\frac{4}{3}\left[\frac{\alpha_{B}}{\alpha} \frac{m_{\Upsilon}^{2}}{m_{B}^{2}-m_{\Upsilon}^{2}}+\left(\frac{\alpha_{B}}{\alpha}\right)^{2}\left(\frac{m_{\Upsilon}^{2}}{m_{B}^{2}-m_{\Upsilon}^{2}}\right)^{2}\right],
$$

where $\alpha$ is the fine-structure constant. This result includes the interference with s-channel photon-exchange [13]. The measured QCD coupling from $\Upsilon$ decay is $\alpha_{s}\left(m_{Z}\right)=0.108 \pm 0.010$ [9], which implies $\Delta R_{\Upsilon}<17.2$ at two standard deviations. The resulting constraint on the free parameters of our model is $m_{B}>m_{\Upsilon} \sqrt{1+43.8 \alpha_{B}}$ which is shown in Fig 1. For the case where $m_{B}<m_{\Upsilon}$, the same argument gives us the constraint $m_{B}<m_{\Upsilon} \sqrt{1-33.2 \alpha_{B}}$, also shown in Fig. 1. For $m_{B}<m_{\Upsilon}$, another possible constraint comes from the decay $\Upsilon \rightarrow \gamma_{B} g g$. In the limit $m_{B} \approx 0$, we obtain the limit $\alpha_{B}<0.149$. Since this constraint is weaker than those discussed above we have not shown it in Fig. 1. The CP-even excited states $\chi_{b 0}, \chi_{b 1}, \chi_{b 2}$ can decay into a real $\gamma \gamma_{B}$ final state, so that a search for a monochromatic photon may also exclude some portion of the parameter space. This requires a more careful study, and will be discussed elsewhere [8].

If $m_{B} \gtrsim 20 \mathrm{GeV}$, all analyses of $\alpha_{s}$ based on deep inelastic scattering data, the lattice QCD calculations of the quarkonia spectrum, and the $\tau$ hadronic decay width, will remain unaffected by the existence of the $\gamma_{B}$. It is worth pointing out that these measurements tend to give smaller values of $\alpha_{s}$ compared to the value extracted from measurements made at LEP, in particular, from the measurement of $\Gamma(Z \rightarrow$ hadrons $)$. Since the $\gamma_{B}$ boson provides an additional positive contribution to $\Gamma(Z \rightarrow$ hadrons $)$, the data may be viewed as suggesting its existence [14]. However, since the various measurements of $\alpha_{s}\left(m_{Z}\right)$ seem to be converging, we feel that it is a more conservative approach to restrict the parameter space of our model based on the experimental data, rather than to predict specific experimental anomalies.

Finally, we discuss signatures of the $\gamma_{B}$ boson that might be discerned by further study of existent accelerator data. In recent analyses of four-jet 
events [15], the QCD group theory factors $N_{C}$ and $T_{F}$ were fit using the $\theta_{B Z}$ and $\theta_{N R}$ distributions [16]. The $\gamma_{B}$ contribution leads to an enhancement in the number of $q \bar{q} q \bar{q}$ final states, similar to the signature of an abelian gluon. The fits allow $T_{F}$ to be roughly twice as large as the QCD prediction, which would allow us to exclude the region above $\alpha_{B} \simeq 0.1$ if $y_{\text {cut }} \simeq m_{B}^{2} / m_{Z}^{2}$ (see Fig. 3). However, the results in Ref. [15] for $y_{\text {cut }}=0.01-0.03$ correspond to $m_{B}$ in the range $9-16 \mathrm{GeV}$, which is already excluded down to $\alpha_{B} \approx 0.04$ by the constraints from $\Upsilon$-decay. Thus, the data must be re-analyzed for larger $y_{\text {cut }}$ (up to $\approx 0.12$ ) before we can put further constraints on the $m_{B}-\alpha_{B}$ plane. The absence of a next-to-leading order calculation of the QCD background, and the lower statistics at higher $y_{\text {cut }}$ will present the main problems in this analysis.

Perhaps the most interesting potential signal of the $\gamma_{B}$ boson is events with large rapidity gaps $\Delta \eta_{c}$ in hadronic collisions, which are expected when scattering proceeds by color-singlet exchange. At the Tevatron, rapidity gap events have been searched for at $E_{T}>30 \mathrm{GeV}$ and $\Delta \eta_{c}>3$. In the large gap limit $\left(\Delta \eta_{c} \gtrsim 4\right)$, two-jet events are dominated by $q \bar{q}$ scattering via gluon exchange because the center-of-mass energy of the subprocess grows exponentially with $\Delta \eta_{c}, \sqrt{\hat{s}}=2 E_{T} \cosh \Delta \eta_{c} / 2$. The ratio of the events via $\gamma_{B}$ exchange to those by gluon exchange is $\left(\alpha_{B}^{2} / 18 \alpha_{s}^{2}\right)\left(1+m_{B}^{2} / E_{T}^{2}\right)^{-2}$. Given an estimate of the survival probability of the rapidity gap, $S \simeq 0.1-0.3$ [17], the contribution of $\gamma_{B}$ exchange to the rate of events with a large rapidity gap is

$$
f\left(\Delta \eta_{c}>4\right) \sim(0.1-0.3) \frac{4 \times 10^{-2}}{\left(1+m_{B}^{2} / E_{T}^{2}\right)^{2}}\left(\frac{\alpha_{B}}{0.1}\right)^{2} .
$$

The rate is remarkably close to the experimental observations [5] when $\alpha_{B} \simeq$ 0.1 and $m_{B} \lesssim 30 \mathrm{GeV}$. While it has been suggested that the data could be explained by the exchange of a QCD pomeron [18], it is tempting to speculate that $\gamma_{B}$ exchange might instead be the origin of the events with large rapidity gaps [19].

Finally we present one simple extension of the Standard Model in which $\mathrm{U}(1)_{B}$ is gauged in an anomaly-free way. To gauge the baryon number current, we need to introduce additional fermions to cancel the $\mathrm{U}(1)_{B} \mathrm{SU}(2)_{L}^{2}$, $\mathrm{U}(1)_{B} \mathrm{U}(1)_{Y}^{2}, \mathrm{U}(1)_{B}^{2} \mathrm{U}(1)_{Y}$ and $\mathrm{U}(1)_{B}^{3}$ triangle anomalies. To do so, we introduce $N_{Q}$ families each consisting of an $\mathrm{SU}(2)$ doublet of left-handed fermions 
$Q_{L}=\left(U_{L}, D_{L}\right)$ with zero hypercharge, and two $\mathrm{SU}(2)$ singlet right-handed fermions, $U_{R}$ and $D_{R}$ with hypercharges $1 / 2$ and $-1 / 2$, respectively. We assume that these new fermions acquire degenerate Dirac masses from electroweak symmetry breaking (so that there will be no contribution to the $T$ parameter). Assuming a common baryon number $B_{Q}$ for each of these fields, all anomalies cancel when $B_{Q} N_{Q}=-3$. [20]. It is noteworthy that this particle content is exactly what is found in a minimal one-doublet technicolor model [21], with or without fundamental scalars [22]. The constraint from the $S$-parameter [23] $S_{\text {new }}=N_{Q} /(6 \pi)<0.46(95 \%$ C.L. [9]) can be easily met when $B_{Q} \gtrsim 0.35$.

Since we have assumed that the $\gamma_{B}$ boson becomes massive through spontaneous symmetry breakdown, there is also an associated Higgs boson. However, since we do not know the Higgs boson's baryon number $B_{H}$, or its quartic self-coupling $\lambda$, we cannot predict its mass $\sim \lambda m_{B} /\left(\sqrt{4 \pi \alpha_{B}} B_{H}\right)$. Notice that if we let $B_{H} \rightarrow 0$, we can make the Higgs mass arbitrarily large. If we assume $B_{H}=1 / 3$ and $\lambda \approx 1$, then the mass of the Higgs boson will be around the $100 \mathrm{GeV}$ scale. The Higgs bosons decays into a real $\gamma_{B} \gamma_{B}$ pair, and thus, to four jets. It can be copiously produced by $\gamma_{B}$ fusion in $q \bar{q}$ collisions or by the Bjorken-like process $q \bar{q} \rightarrow \gamma_{B}^{*} \rightarrow \gamma_{B} H$, but the final state is completely hadronic, and the signal is difficult to see. It is important to note that the baryon number current is still conserved even after the spontaneous breakdown of $\mathrm{U}(1)_{B}$. Therefore there is no constraint from proton decay experiments.

Conclusions. We have shown that a new light $\mathrm{U}(1)$ gauge boson $\gamma_{B}$ coupled to the baryon number is consistent with all existing experimental constraints. The allowed region of the model's parameter space corresponds roughly to $m_{B} \gtrsim 20 \mathrm{GeV}$ and $\alpha_{B} \lesssim 0.2$. We have pointed out that the rapidity gap events observed at Tevatron may be a manifestation of $\gamma_{B}$. We have also shown that the gauge anomalies can be canceled easily by introducing a small number of new fermions, with exactly the same quantum numbers as in a minimal one-doublet technicolor model.

\section{Acknowledgments}

We are grateful to Mike Barnett, Lawrence Hall, Ian Hinchliffe, and Axel Kwiatkowski for useful comments. This work was supported by the Direc- 
tor, Office of Energy Research, Office of High Energy and Nuclear Physics, Division of High Energy Physics of the U.S. Department of Energy under Contract DE-AC03-76SF00098.

\section{References}

[1] S. Giddings and A. Strominger, Nucl. Phys. B307, 854 (1988); S. Coleman, Nucl. Phys. B310, 643 (1988); G. Gilbert, Nucl. Phys. B328, 159 (1989).

[2] See for instance, A.E. Faraggi, Nucl. Phys. B428, 111 (1994); H. Murayama and D.B. Kaplan, Phys. Lett. B336, 221 (1994); V. Ben-Hamo and Y. Nir, WIS-94-34-PH, hep-ph/9408315.

[3] See e.g., C.N. Yang and R.L. Mills, Phys. Rev. 96, 191 (1954).

[4] There is also a parameter that describes the kinetic mixing between the $\mathrm{U}(1)_{B}$ and $\mathrm{U}(1)_{Y}$ gauge bosons (see e.g., A. Nelson and N. Tetradis, Phys. Lett. B221, 80 (1989)). LEP data and low-energy $e^{+} e^{-}$experiments constrain this parameter to be small, and we do not discuss it any further here. Phenomenology associated with the kinetic mixing will be considered in 8 .

[5] D0 Collaboration (S. Abachi et al.), Phys. Rev. Lett. 72, 2332 (1994); CDF Collaboration (F. Abe, et al.), FERMILAB-PUB-94-194-E.

[6] For a comprehensive review, see H.E. Haber, SCIPP-93-21, hepph/9308235, and references therein. For constraints from LEP, see G.R. Farrar, Phys. Lett. B265, 395 (1991); R. Muñoz-Tapia and W.J. Stirling, Phys. Rev. D49, 3763 (1994).

[7] T. Kon and T. Nonaka, Phys. Lett. B319, 355 (1993); M. Fukugita, H. Murayama, M. Yamaguchi, and T. Yanagida, Phys. Rev. Lett. 72, 3009 (1994).

[8] C.D. Carone and H. Murayama, work in progress.

[9] Particle Data Group (L. Montanet et al.), Phys. Rev. D50, 1173 (1994). 
[10] All the distributions are computed by HELAS (H. Murayama, I. Watanabe, and K. Hagiwara, KEK-91-11) and BASES (S. Kawabata, Comput. Phys. Commun. 41127 (1986)).

[11] OPAL Collaboration (M.Z. Akrawy et al.), Z. Phys. C 49, 49 (1991).

[12] ALEPH (D. Decamp et al.), Phys. Rep. 216, 253 (1992)

[13] The running of $\alpha_{B}$ from $m_{Z}$ to $m_{\Upsilon}$ is small for $\alpha_{B} \lesssim 0.2$, allowed by the $Z$ hadronic width.

[14] This point was noted independently by David Bailey and Sacha Davidson, private communication.

[15] ALEPH Collaboration (D. Decamp et al.), Phys. Lett. B284 151 (1992);

L3 Collaboration (B. Adeva et al.), Phys. Lett. B248 227 (1990); DELPHI Collaboration (P. Abreu et al.), Z. Phys. C 59, 357 (1993); OPAL Collaboration (R. Akers et al.), CERN-PP3/94-135.

[16] O. Nachtmann and A. Reiter, Z. Phys. C 16, 45 (1982); M. Bentsson and P.M. Zerwas, Phys. Lett. B208, 306 (1988).

[17] E. Gotsman, E.M. Levin, and U. Maor, Phys. Lett. B309, 199 (1993).

[18] W.J. Stirling, Nucl. Phys. B 423, 56 (1994); H.N. Chehime and D. Zeppenfeld, MAD-PH-814, hep-ph/9401244.

[19] For $m_{B}<m_{\Upsilon}$, there is also some contribution to the rapidity gap events at HERA [8].

[20] In addition, $N_{Q}$ must be taken to be even to avoid the $\mathrm{SU}(2)$ anomaly (E. Witten, Phys. Lett. B117, 324 (1982)).

[21] S. Weinberg, Phys. Rev. D13, 974 (1976); Phys. Rev. D19, 1277 (1979); L. Susskind, Phys. Rev. D20, 2619 (1979).

[22] For one example of a technicolor model with scalars, see C.D. Carone and H. Georgi, Phys. Rev. D49, 1427 (1994).

[23] M.E. Peskin and T. Takeuchi, Phys. Rev. Lett. 65, 964 (1990). 


\section{Figure Captions}

Fig. 1. Allowed regions of the $m_{B}-\alpha_{B}$ plane at $95 \%$ C.L. or two standard deviations. The bounds shown come from (1) the $Z$ hadronic width, (2) the fraction of four-quark jet events in four-jet events, (3) the hadronic decay width of the $\Upsilon(1 S)$. The parameter space above each of the lines shown is excluded, and the weaker constraints discussed in the text are not shown.

Fig. 2. Four-jet cross section as a function of $y_{\text {cut }}$ for $\alpha_{B}=0.1$, normalized to the leading two-jet cross section.

Fig. 3. Di-jet invariant mass distribution in four-jet events, for $\alpha_{B}=0.1$ and $y_{\text {cut }}=0.04$, normalized to the leading two-jet cross section.

Fig. 4. Four-jet differential cross section as a function of $y_{\min }$ for $\alpha_{B}=$ 0.1 , normalized to the leading two-jet cross section. 


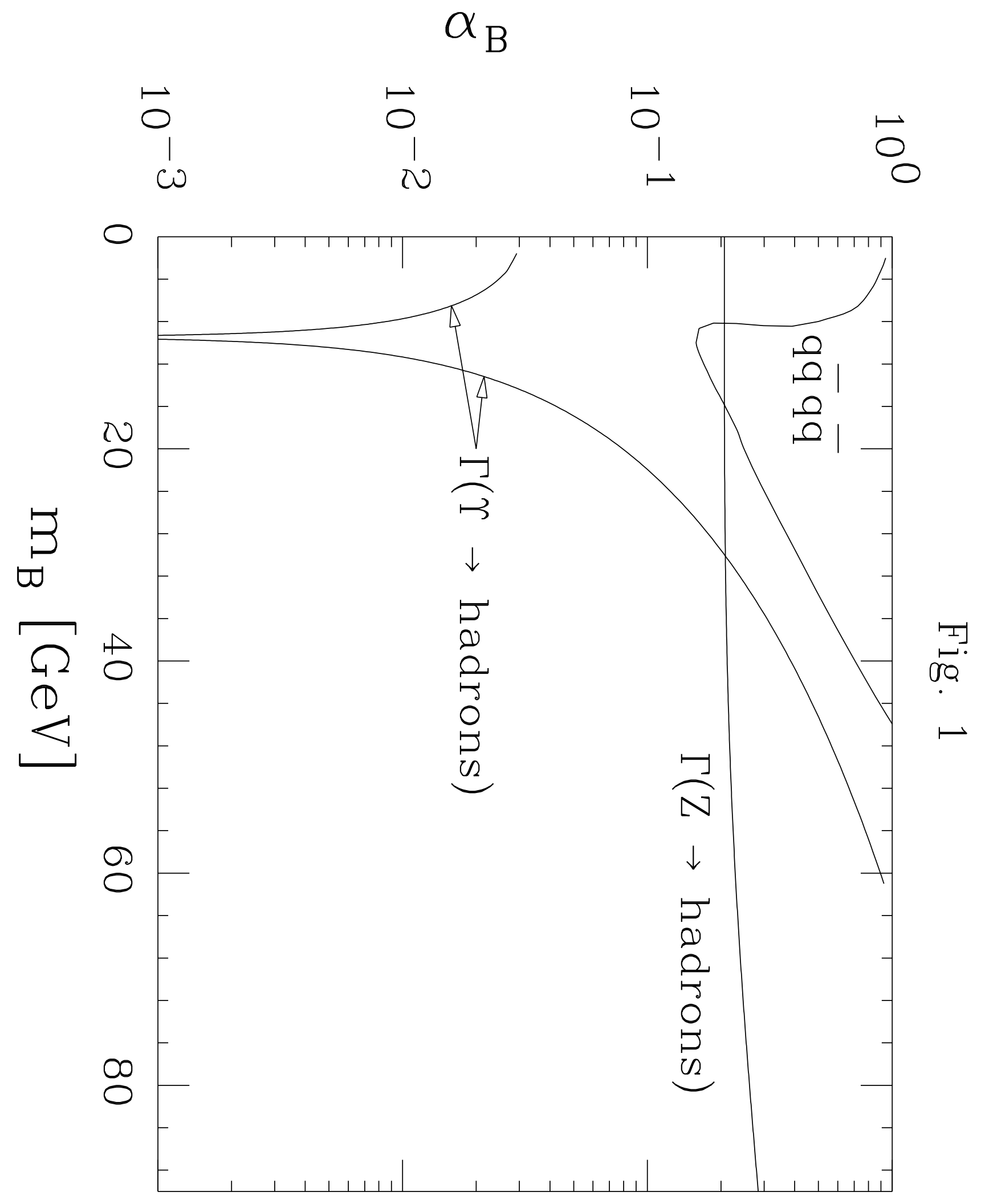


This figure "fig1-1.png" is available in "png" format from: http://arxiv.org/ps/hep-ph/9411256v2 
This figure "fig1-2.png" is available in "png" format from: http://arxiv.org/ps/hep-ph/9411256v2 
This figure "fig1-3.png" is available in "png" format from: http://arxiv.org/ps/hep-ph/9411256v2 
This figure "fig1-4.png" is available in "png" format from: http://arxiv.org/ps/hep-ph/9411256v2 


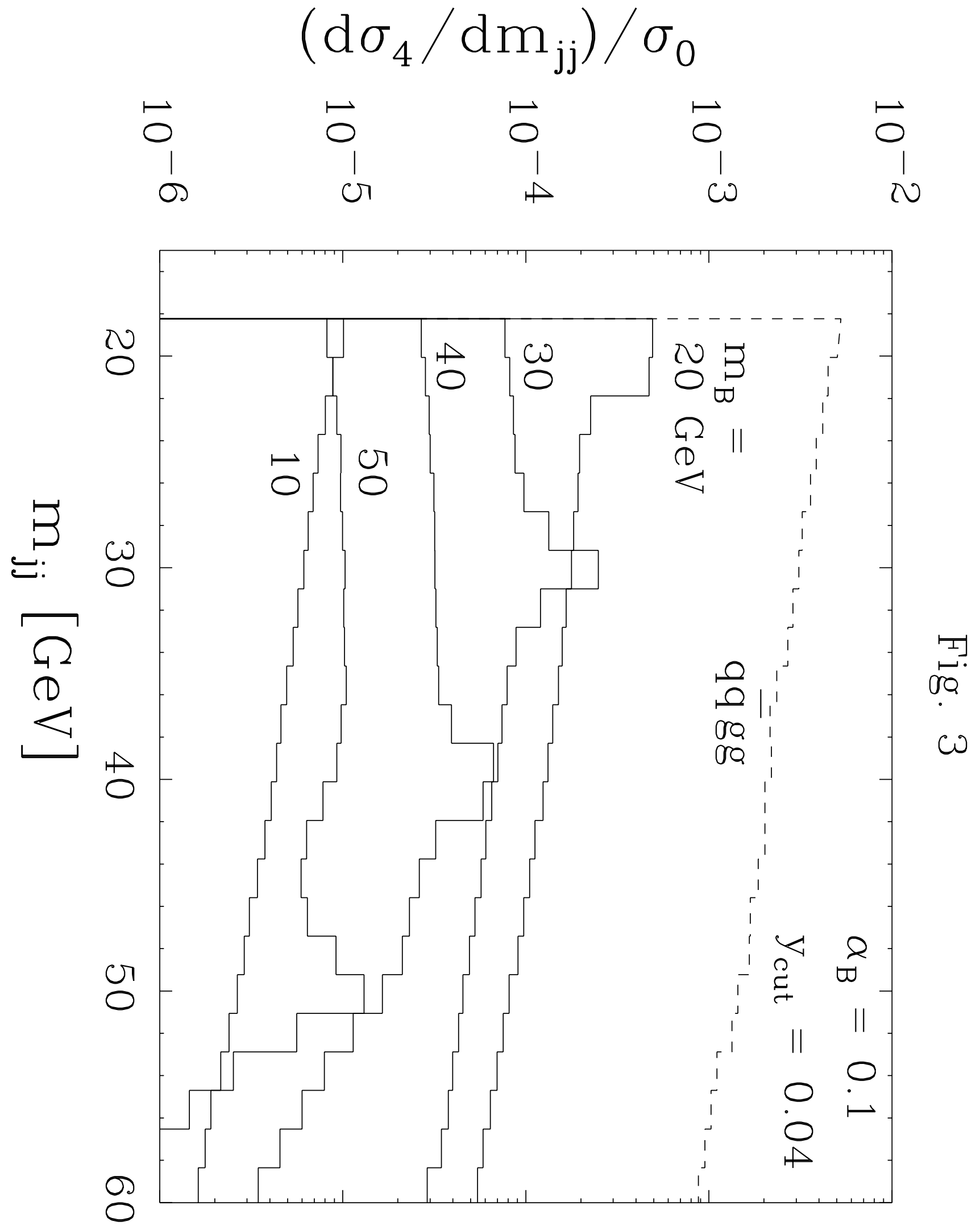




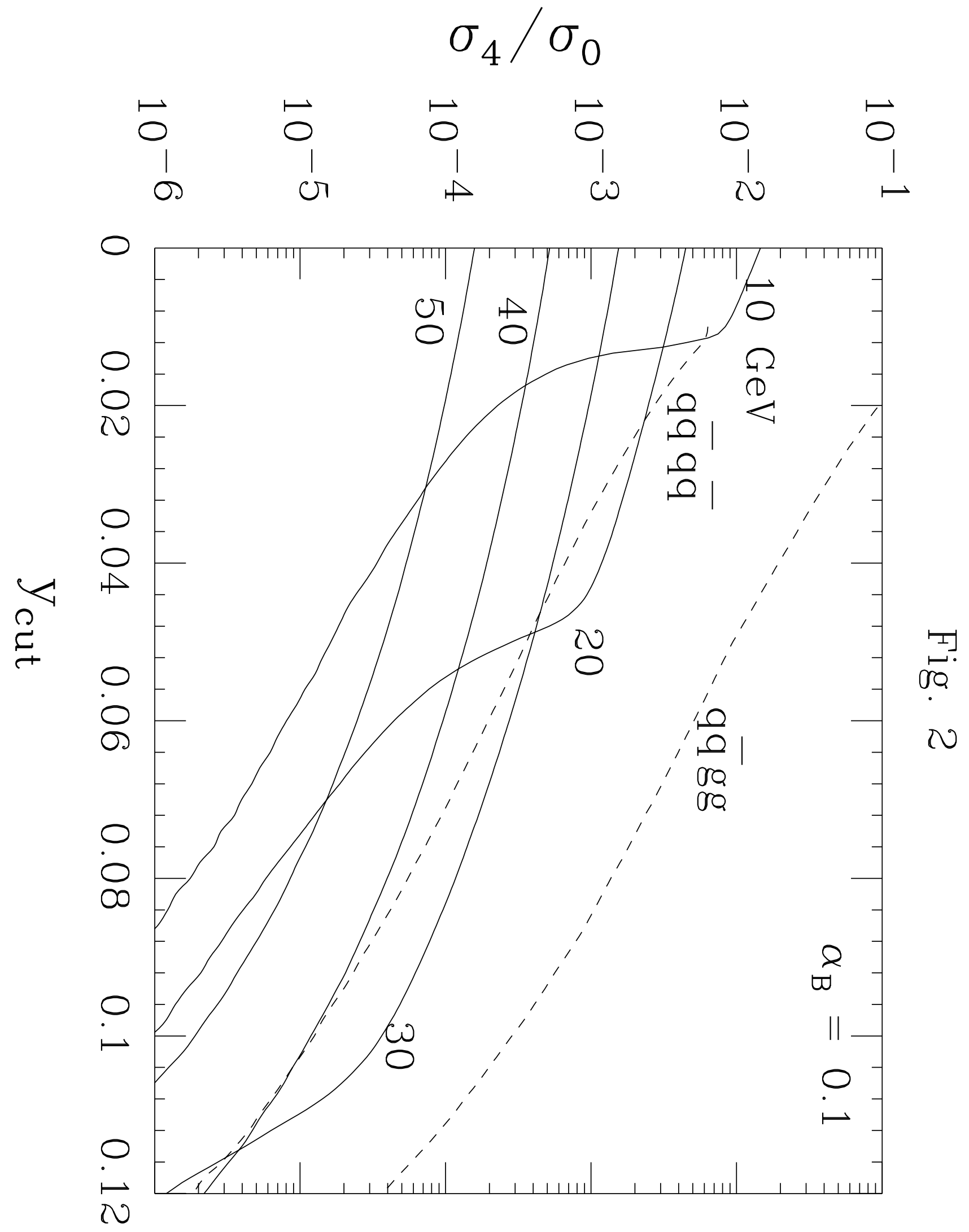




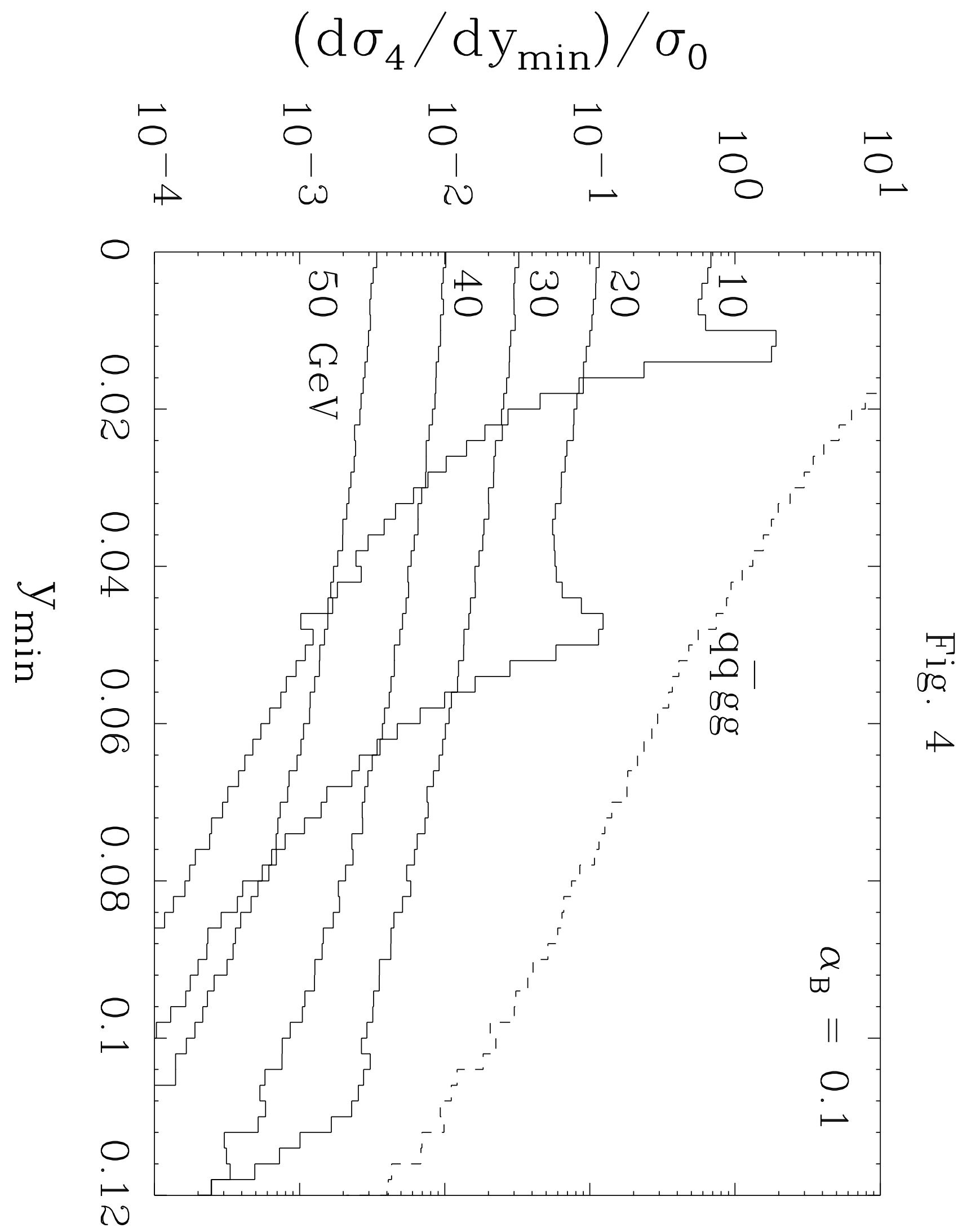

